\title{
Development of a Low Cost Point Absorber Wave Energy Converter for
} Electric Mobility

\author{
Jacob W. Foster ${ }^{1}$, Reza Ghorbani ${ }^{1}$, Pierre Garambois ${ }^{2}$, Emma Jonson $^{3}$, Sten Karlsson ${ }^{3}$ \\ ${ }^{1}$ University of Hawai $i$ at Manoa, Honolulu, USA; \\ ${ }^{2}$ INSA, Lyon,France; \\ ${ }^{3}$ Chalmers Univ. of Technology, Gothenburg, Sweden \\ *Corresponding author: Tel 713-419-666; E-mail: fosterja@hawaii.edu
}

\begin{abstract}
This paper presents a developing concept of a low cost "point absorber" Wave Energy Converter (WEC). The WEC is unique in that instead of operating with one buoy, two are used to optimize the hydrodynamic response and energy output. This makes the design a three-part system: the surface buoy provides the vertical translation and hosts the primary interface for energy absorption, the power take-off (PTO) device which is a direct drive permanent magnet AC generator, and lastly the tension buoy that feeds through the PTO to the surface buoy. The total cost of the $2 \mathrm{KW}$ WEC is less than \$2000 USD including the PMAC generator, power converter and cable to charge the batteries for electric vehicles. The integrated dynamics of the WEC and the PTO are presented using a simplified model of a heaving buoy. The wave energy resource of a test location is analyzed and presented based on data from a global Wave Watch III model surrounding Oahu. The characteristics and distributions of the movement patterns of individual vehicles are measured. It was shown that regenerative braking power has the major role in reducing the total energy consumption and decreasing the size of the required battery to be charged externally by the WEC. In addition the power used by vehicle follows the Rayleigh distribution. Thus, the batteries for individual driver are customizable. This significantly reduces the amount of energy required by distributed WEC generators.
\end{abstract}

Keywords: Wave energy conversion, point absorber, electric vehicle

\section{Introduction}

Throughout the development of Wave Energy Conversion devices theoretical solutions have been produced for various methods of energy extraction for both point absorbing buoys and oscillating water column devices. Analytical solutions are formulated from the hydrodynamic interaction between the device interfaces with incoming waves (see Evans 1981, Newman 1979; McCormick 1980, Cruz 2008, Garnaud and Mei 2010).

As WEC technologies have evolved some commercial entities have taken the stage in pursuing industrial development of these devices for large scale energy production (Clément et al. 2002). Care is taken into selecting design criteria based on hydrodynamic and powertake-off (PTO) parameters. It is of use to identify these criteria so as to perform a wave climate-wise optimization. This paper presents the design of a wave-energy conversion device and a brief overview of the wave-climate at the location where the device is to be tested. The characteristics and distributions of the movement patterns of individual vehicles are measured. It was shown that regenerative braking power has the major role in reducing the total energy consumption and decreasing the size of the required battery to be charged externally by the WEC. In addition the power used by vehicle follows the Rayleigh distribution. Thus, the batteries for individual driver are customizable. This significantly reduces the amount of energy required by distributed WEC generators.

\section{Methodology}

To develop a general equation for the power capture capability of this device the analytical solution of a small point absorber, as presented by Garnaud and Mei (2010), is discussed and extended for the WEC device in this section. A wave climate analysis is provided to present 
base climate criteria of which control variables must be optimized for WEC. The site selected is located off the North East coast of the Island of Oahu where trade-wind seas are predominant.

\subsection{Power extraction concept:}

The premise for the WEC device is the uniqueness of the power-take-off system. The PTO unit is sealed and anchored to the sea-floor. Housed within are the motor/generator and inertial wheel which are driven via an external capstan (see Fig.1). The design is based on a dry PTO unit. In this setup the PTO is not sealed watertight to be submerged and anchored to the sea-floor (see Fig. 2.). Rather, it is suspended safely above the sea-surface via a column fastened to the concrete anchor-base.

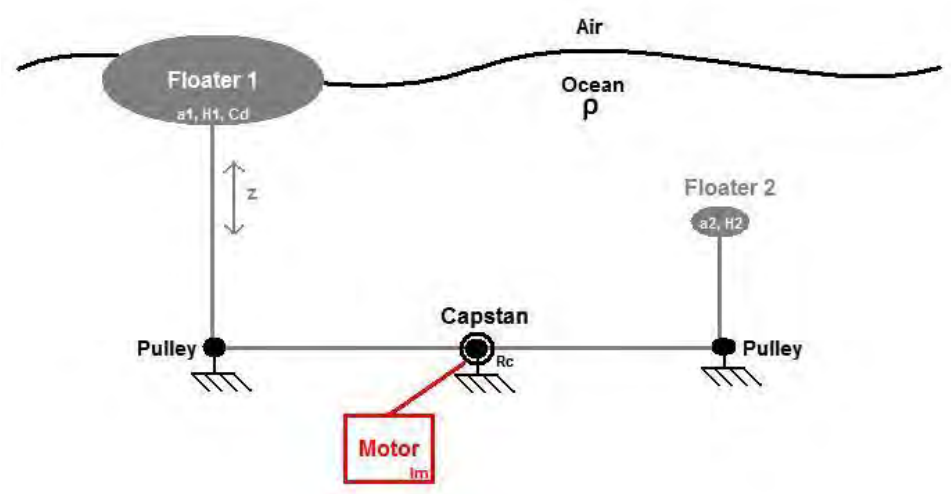

Fig. 1. Schematic of the three-part WEC device

The PTO capstan is the primary interface between the two buoys. Through it, the vertical buoy motion due to forcing from incoming waves is transferred to rotational motion to be resisted by the motor/generator and inertial wheel. While the larger-floating buoy acts as a point absorber with incoming waves the smaller submerged buoy provides the required tension to resist slipping between the tension line and the capstan. The tension relationship is defined as:

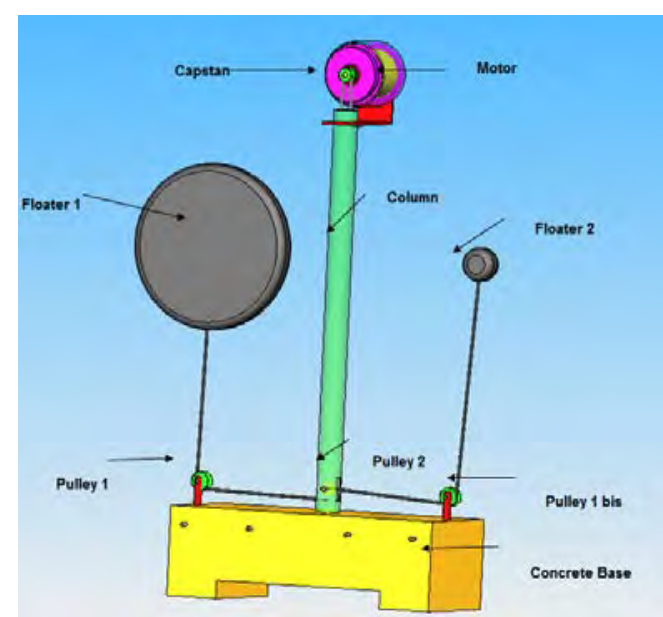

Fig. 2. Dry PTO WEC design

$$
T_{2}=T_{1} e^{\mu^{*} \text { Around }},
$$

where, $\mu=0.4$ (the friction coefficient between rope and steel) and $\mathrm{T}_{1} ; \mathrm{T}_{2}$ are the tension on the line approaching the point-absorber and the tension buoy respectively.

Through investigation we find that the required size of tension buoy is significantly smaller than that of the point-absorber. With this in mind we begin to develop the general equation for the average power extraction of the three-part WEC device.

The following formulation for such a solution is an extension of the analytical solution presented by Garnaud and Mei (2010) for a single-small point absorber. To demonstrate the performance of a single degree of freedom system, a buoy responding due to the vertical heave forcing of a wave (with angular frequency $\omega$ and amplitude A) is considered. Incoming wave potential is defined as 


$$
\Phi_{I}=\varphi_{I} e^{-i \omega t}
$$

with

$$
\varphi_{I}=\frac{A g}{i \omega} \frac{\cosh (k(z+h))}{\cosh (k h)} e^{i k x}
$$

where $\omega$ and $\mathrm{k}$ are related by the dispersion relation

$$
\omega^{2}=g k \tanh (k h) .
$$

At this point Garnaud and Mei (2010) make a simplification based on the small size of the surface buoy relative to incoming wave length, in that the scattered and radiated waves are negligible. This is from the Froude-Krylov approximation where the hydrodynamic pressure on the buoy is dominated by the undisturbed incoming wave (Newman 1979). The vertical excitation force on a buoy of radius, a, and draft, $\mathrm{H}$, becomes

$$
i \rho \omega \int \quad \int(0,0,0), d S=\rho g A \pi a^{2}
$$

We now include the power-take-off influence of the 3-part device to the general WEC equation by assuming the PTO exerts a load of $\omega^{2} \lambda_{\mathrm{g}} \zeta$ where $\lambda_{\mathrm{g}}$ is the extraction rate, and $\zeta$ is the buoy displacement. The force due to the inertial wheel can be included as $\frac{I^{*} \ddot{\varsigma}}{R_{c}}$, where $I$ is the inertia the drive system, and $R_{c}$ is the radius of the capstan. The added buoyancy force on the floating buoy, due to heave is $\pi a_{1}^{2} \rho g \varsigma$ then by Newton's law

$$
\left(M_{1} \varsigma+M_{2} \varsigma+\frac{I \varsigma}{R^{2}{ }_{c}}\right) \omega^{2}+\lambda_{g} \varsigma i \omega+\pi \rho g a_{1}^{2} \varsigma=\pi \rho g a_{1}^{2} A,
$$

where $M_{1}$ and $M_{2}$ can be defined from Archimedes principle as:

$$
M_{1}=\rho \pi a_{1}^{2} H_{1}
$$

and

$$
M_{2}=\rho \pi a_{2}^{2} H_{2} / 2 \text {. }
$$

From this we can define a transfer function for amplitude of response as

$$
\frac{\varsigma}{A}=\frac{1}{\left\{\frac{H_{1}}{g}+\frac{H_{2}}{2 g}\left(\frac{a_{1}}{a_{2}}\right)^{2}+\frac{I}{R_{c}^{2} \rho \pi a_{1}^{2}}\right\} \omega^{2}+\frac{\lambda_{g}}{\rho \pi a_{1}^{2} g} i \omega+1}
$$

This lets us define the time average rate of energy extraction at a single frequency as 


$$
P_{\text {buoy }}=\overline{\lambda_{g}\left[e\left(\varsigma e^{i \omega t}\right)\right]^{2}}=\frac{1}{2} \omega^{2} \lambda_{g}|\varsigma|^{2}
$$

or, for an irregular time series of multiple frequencies

$$
P_{\text {buoy }}=\frac{1}{2} \lambda_{g}|\dot{\zeta}|^{2}
$$

\subsection{Wave climate and optimization schemes}

The data for the climate-wise optimization was provided by (Arinaga and Cheung 2011 and Stopa and Cheung 2011). Ten years of wave hindcast parametric spectral data based on significant wave height, $\mathrm{H}_{\mathrm{s}}$, and peak period, $\mathrm{T}_{\mathrm{P}}$, were generated using a global Wave Watch III simulation with a nested output for the Hawaiian Islands. (An explanation of wave power spectrum can be found in Cruz, 2008). Fig. 2 depicts this data in a multivariate histogram displaying the frequency of occurrence in color of a sea-state with parameters $H_{s}, T_{P}$ along the vertical and horizontal axis.

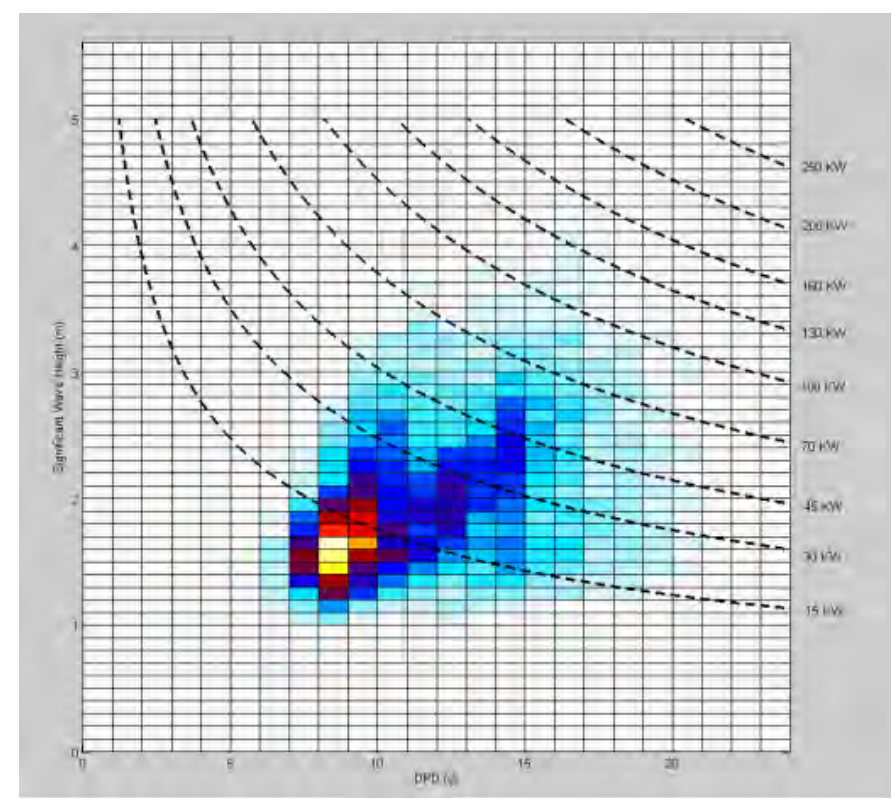

Fig 2. Multivariate histogram of sea-state occurrence with incident wave power contour lines. The horizontal and vertical axis relate to the peak period and significant wave height of a six-hour seastate respectively. The frequency of occurrence is shown in color, and contour lines depict wave power per sea-state at the associated Tp and Hs per the Bretschneider spectrum approximation.

The contour lines represent the average power per energy period within a sea-state at the associated $\mathrm{H}_{\mathrm{s}}, \mathrm{T}_{\mathrm{P}}$ value and are given by:

$$
P_{o}=\rho g^{2} \frac{H_{s}^{2} T_{e}}{64 \pi}
$$

This formulation is derived from the statistical moments of a wave power spectrum. For a Bretschneider spectrum 
$S(\omega)=\frac{5}{16} H_{s}{ }^{2} \frac{\omega_{p}{ }^{4}}{\omega^{5}} e^{-5 / 4\left(\frac{\omega_{p}}{\omega}\right)^{4}}$

the following relationship can be defined:

$T_{e}=2 \pi \frac{m_{-1}}{m_{o}}=0.857 * T_{P}$

Where $m_{-1}, m_{\odot}$ are spectral moments of the curve, $S(\omega)$. The wave amplitude for any given angular frequency can be found from

$A=\sqrt{2 * S(\omega) * \omega}$.

The Capture Width of a device is used evaluate a WEC's performance at any given frequency. This is the ratio of the total mean power absorbed by the WEC to the mean power per unit wave crest width of the incident wave train;

$l(\omega)=\frac{P_{\text {buoy }}}{P_{o}}$

Since the incident wave power is per unit wave crest width, the absorption width is length dimensional (and ideally greater than the width of the device itself). The total incident wave power in a single sea-state, $S(\omega)$, is given as

$P_{T}=\rho g \int_{0}^{\infty} S(\omega) c_{g}(\omega) d \omega$

where

$$
c_{g}(\omega)=g / 2 \omega
$$

for deep water, as derived from the dispersion relationship. The power absorption capability in a sea-state can be computed by multiplying the capture width of a WEC at each frequency in the above integral to obtain the total absorbed power. This is defined as

$$
P_{T}=\rho g \int_{0}^{\infty} S(\omega) c_{g}(\omega) l(\omega) d \omega
$$

Since $P_{T}=\mathrm{f}\left(\mathrm{H}_{\mathrm{s}}, \mathrm{T}_{\mathrm{P}}\right)$ for each sea-state, a long-term analysis for a single site requires the use of the multivariate histogram to compute the probability of occurrence of sea-states, $S(\omega)$, which may occur during the operational lifetime of the WEC. With this knowledge in hand one can begin to identify variable design parameters to be optimized for device operation. We allow $\lambda_{\mathrm{g}}$ to be the free variable for power extraction optimization since the extraction rate can be controlled by power delivery mechanisms. If the desire is to optimize the WEC system in a "robust" manner (i.e. that it is suited for a range of sea-states which may occur over the lifetime of the device) this variable is optimized for maximum average power extraction 
according to Eq. (19) where the capture width $l(\omega)$, by definition, carries $\lambda_{\mathrm{g}}$ from Eq. (10) and $\mathrm{Eq}(16)$.

However, in the interest of fine-tuning the device response for individual incoming sea-states, which may be predicted by meteorological and wave forecast methods, the optimization will be performed on Eq. (11) alone, such that an optimum value of $\lambda_{\mathrm{g}}$ can be found at each frequency $(\omega)$ of a wave power spectrum Eq. (13). To illustrate the latter optimization scheme the generator extraction rate, $\lambda_{\mathrm{g}}$, was set to values of 1000,5000 and $10000 \mathrm{~kg} / \mathrm{s}$ respectively.

\section{Results}

\subsection{Wave Buoy Response Simulation}

The buoy response was computed in the time domain utilizing the time series simulation platform Simulink from MATLAB. The response transfer function, Eq. (9), was directly applied to a simulated 200 second time series wave-record which was generated by discretizing a Bretschneider Spectrum Eq. (13). The numerical spectrum was formed with a significant wave height was 1.5 meters and peak period of 9 seconds to agree with the typical sea-states at the selected site. Following Eq. (11) the average power extraction of the device is computed, and then by simple integration the energy is calculated and presented. As expected the buoys response experiences a lag of the incoming wave motion and varies significantly based on the selected extraction rate as shown in Fig. 4.

\subsection{Electric Vehicle Power Study}

A Swedish car movement data project started in June 2010 in the Västra Götaland county, going on until June 2011. The aim is to gather a larger amount of data on the characteristic and distribution of the movement patterns of individual, privately driven cars in Sweden by measurement with gps equipment.

We use data from six cars in the Swedish car movement data project, each driving one day, to investigate the probability distribution of the momentary power consumption. The propulsion is equal to the sum of the aerodynamic drag, frictional forces and the acceleration force. The potential energy from regenerative braking, i.e. the integral of negative power, is, based on this statistics data, greater than $50 \%$ of the propulsion energy (53 MJ compared to 100 $\mathrm{MJ})$. Regenerative braking therefore has good potential for reducing energy use per $\mathrm{km}$ driven. The power could be approximated by a Rayleigh probability density function as shown in Fig. 5. The performance of electric vehicles can be improved by modifying the type of the batteries to be a combination of high-power and high-energy batteries. The high-power batteries improve the gas mileage through regenerative braking and the high-energy batteries can be charged by WEC during opportunity charging. This reduces the required number of charging stations and perhaps the total infrastructural cost associated with electric mobility using distributed energy sources. 


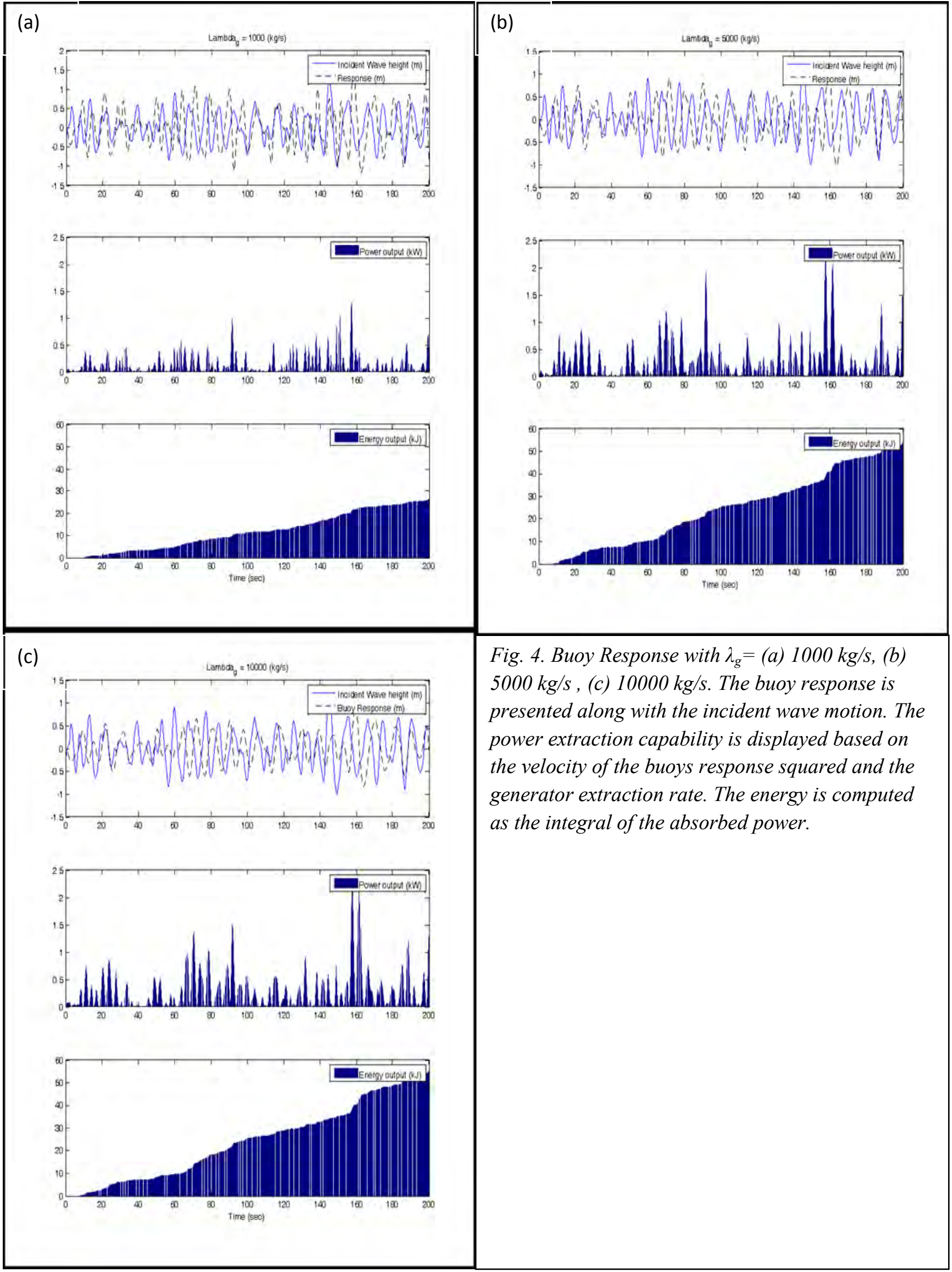



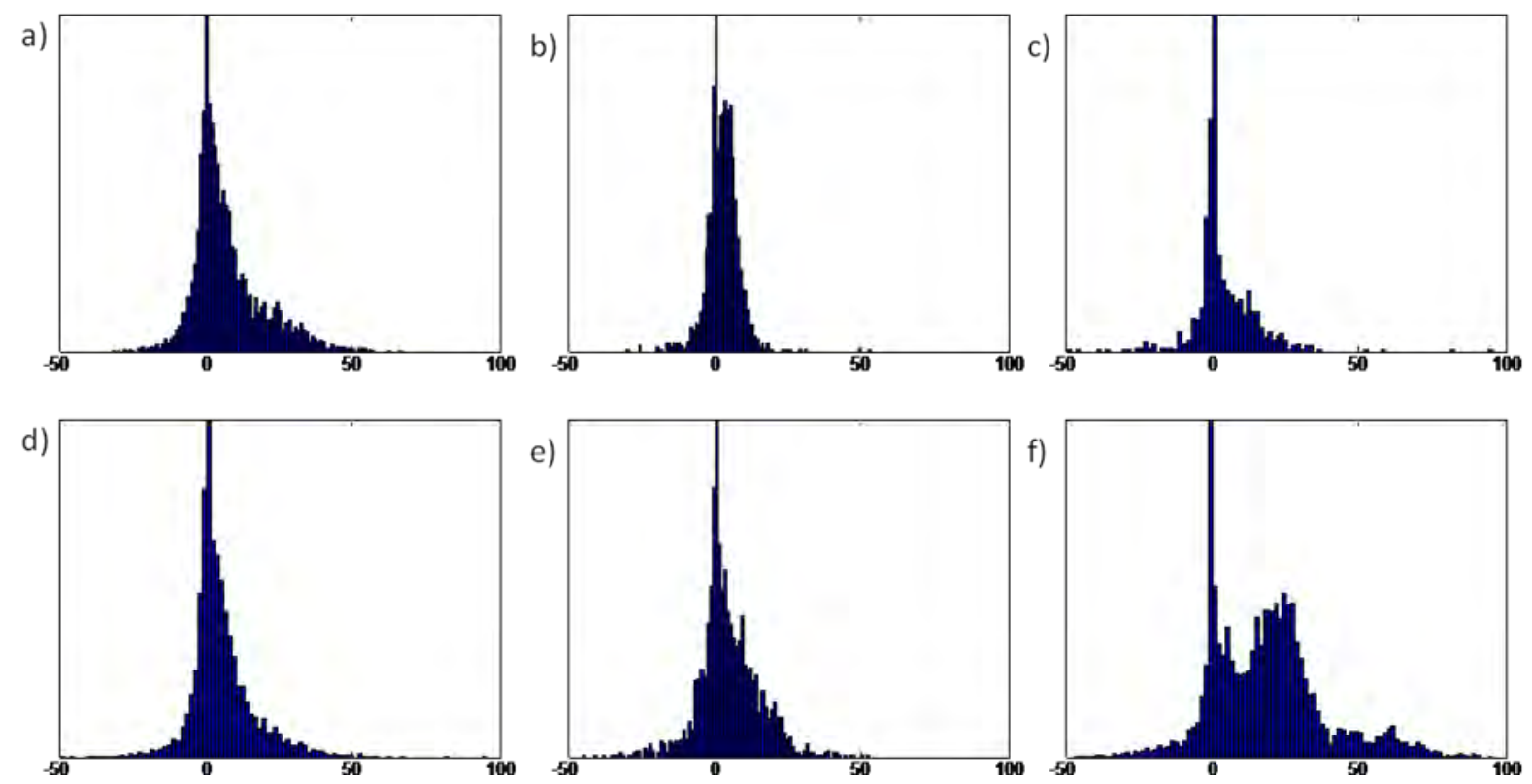

Fig. 5. Probability Histograms

\section{References}

[1] Evans, D. V. "Wave-power Absorption by Systems of Oscillating Surface Pressure Distributions." Journal of Fluid Mechanics 114.-1 (1982): 481-99. Print.

[2] Newman, J. "Absorption of Wave Energy by Elongated Bodies." Applied Ocean Research 1.4 (1979): 189-96. Print.

[3] MacCormick, Michael E. Ocean Wave Energy Conversion. 1981. Print.

[4] Cruz, João. Ocean Wave Energy. Berlin: Springer, 2008. Print.

[5] Garnaud, Xavier, and Chiang C. Mei. "Bragg Scattering and Wave-power Extraction by an Array of Small Buoys." Proceedings of the Royal Society 466.2113 (2010): 79-106. Print.

[6] Cle'ment,, Alain, Pat McCullen,, Antonio Falca o, Antonio Fiorentino, Fred Gardner, Karin Hammarlund, George Lemonis, Tony Lewis, Kim Nielsen, Simona Petroncini, Teresa Pontes, Phillippe Schild, Bengt-Olov Sjo“stro“m, Hans Christian Sørensen, and Tom Thorpe. "Wave Energy in Europe: Current Status and Perspectives." Renewable and Sustainable Energy Reviews 6 (2002): 405-31. Print.

[7] Arinaga, R.A. and Cheung, K.F. Atlas of global wave energy from 10 years of reanalysis and hindcast data. Renewable Energy, in review

[8] Stopa, J.E., Cheung, K.F., and Chen, Y.-L. (2011). Assessment of wave energy resources in Hawaii. Renewable Energy, 36(2), 554-567. 